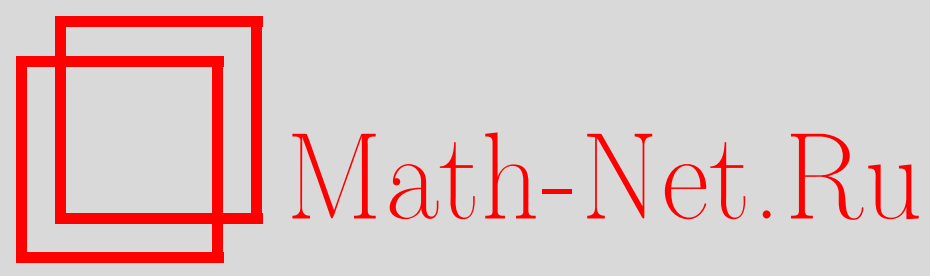

А. В. Боровских, Условия знакорегулярности разрывных краевых задач, Матем. заметки, 2003, том 74, выпуск 5, 643-655

DOI: https://doi.org/10.4213/mzm296

Использование Общероссийского математического портала Math-Net.Ru подразумевает, что вы прочитали и согласны с пользовательским соглашением http://www . mathnet.ru/rus/agreement

Параметры загрузки:

IP : 18.234 .156 .22

26 апреля 2023 г., $07: 04: 22$

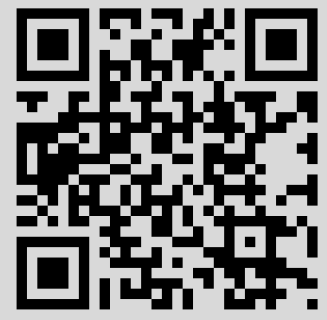




\title{
УСЛОВИЯ ЗНАКОРЕГУЛЯРНОСТИ \\ РАЗРЫВНЫХ КРАЕВЫХ ЗАДАЧ
}

\section{А. В. Боровских}

\begin{abstract}
Для многоточечной разрывной краевой задачи с неосциллируюшим дифференциальным оператором приводится аналог условий Калафати, обеспечивающих свойство знакорегулярности: число перемен знака у решения не превосходит числа перемен знака у правой части. Свойство знакорегулярности позволяет устанавливать для соответствующей спектральной задачи штурмовские свойства спектра (вещественность, положительность, простота собственных значений, перемежаемость нулей собственных функций и т.д.).

Библиография: 23 названия.
\end{abstract}

1. Введение. В известном мемуаре [1] Штурм обнаружил следующие замечательные свойства собственных значений и собственных функций дифференциальных операторов второго порядка специального вида:

1) все собственные значения вещественные, положительные, простые;

2) $i$-я (считая с нуля в порядке возрастания собственных значений) собственная функция имеет точно $i$ нулей;

3) нули $i$-й и $(i+1)$-й функций перемежаются.

Этот комплекс свойств, называемых сейчас осиилляиионными [1]-[14], является одним из наиболее полных и емких качественных описаний, характеризующих общие гармонические свойства колебаний (осцилляций) одномерных упругих систем: он означает, что система колеблется “так же”, как однородная струна. Отличия в числовых значениях собственных частот и в виде функщий, описывающих формы собственных колебаний, не меняют ни структуру спектра в совокупности, ни “внешнего вида" форм собственных колебаний, определяемого количеством и взаимньм расположением узловых точек этих форм.

Основным инструментом обоснования 1)-3) для различных классов спектральных задач вида

$$
L y=\lambda q(x) y, \quad l_{i}(y)=0, \quad i=1, \ldots, n
$$

Результаты, представленные в данной работе, получены при финансовой поддержке Госкомвуза РФ, грант № Е00-1.0-154, Российского фонда фундаментальных исследований, грант № 01-0100418, и программы "Университеты России", проект УР.04.01.047. 
(где $L$ - некоторое обыкновенное дифференциальное выражение, $l_{i}$ - набор функционалов, определяющих краевые условия), являются различные варианты теоремы Келлога-Крейна (см. [2], [6], модификации - [7], [15], [16]) о спектре интегрального оператора

$$
(K y)(x)=\int_{a}^{b} K(x, s) q(s) y(s) d s
$$

с ядром, удовлетворяющим специальным детерминантным неравенствам. Для ядра $K(x, s)$, являющегося функцией Грина соответствующей (1) краевой задачи

$$
L y=f(x), \quad l_{i}(y)=0, \quad i=1, \ldots, n,
$$

эти неравенства сводятся (с несушественными оговорками - см. [7], [15]) к свойству знакорегулярности оператора (2). Это свойство, переформулированное в терминах краевой задачи (3) (мы сохраним за ним тот же термин “знакорегулярность”), состоит в том, что для любой правой части $f(x)$, меняющей знак конечное число раз, соответствующее решение $y(x)$ будет “менее колеблюшимся", чем $f(x)$. Если обозначить число перемен знака функции $f(x)$ через $S(f)$ (точное определение $S(f)$ будет дано чуть позже), а число перемен знака функции $y(x)$ через $S(y)$, то свойство знакорегулярности записьвается в виде неравенства

$$
S(y) \leqslant S(f)
$$

Обоснование знакорегулярности задачи (3) дает, по существу, ключ к получению свойств 1)-3) задачи (1).

Играя ключевую роль в осцилляционной теории, свойство знакорегулярности представляет и самостоятельный интерес, так как “скрьвает” в себе достаточно нетривиальные и тонкие, но совершенно отчетливые свойства. Так, в случае, когда $f(x)$ сохраняет знак (например, $f(x) \geqslant 0$ ) неравенство (4) дает теорему о дифференциальном неравенстве - оно означает, что $y(x)$ также сохраняет знак (который к тому же оказывается - см. [7], [15] - не зависящим от выбора $f(x) \geqslant 0$ ). Если $f_{1}(x)$ монотонна (что эквивалентно неравенству $S\left(f_{1}(x)+C\right) \leqslant 1$ для всех $\left.C \in \mathbb{R}\right)$, то из (4) следует, что для решений $y_{1}(x)$ и $y_{0}(x)$ задачи $(3)$ с правой частью $f_{1}(x)$ и $f_{0}(x) \equiv 1$ соответственно имеет место неравенство $S\left(y_{1}+C y_{0}\right) \leqslant 1$ при любом $C \in \mathbb{R}$, а это эквивалентно монотонности отношения $y_{1} / y_{0}$. Рассмотрение комбинаций из трех функций - константы, линейной и некоторой вьпуклой - показьвает, что решения задачи (3) наследуют, в некотором смысле, и вьпуклость правой части, и так далее.

Настоящая работа посвящена условиям знакорегулярности для разрывных краевых задач. Это класс задач, возникающих, например, при моделировании различных систем из последовательно сочлененных упругих элементов (струн, стержней, пружин и т.п.). Под разрьвной краевой задачей понимается задача отыскания такой функции $y(x)$, непрерьвной и гладкой на $[a, b]$ за исключением конечного набора точек $a<\xi_{1}<\cdots<$ $\xi_{r}<b$ (в которых функция может иметь разрывы и скачки производных), что она удовлетворяет при всех $x \neq \xi_{i}$ некоторому дифференциальному уравнению, в концах отрезка - некоторым краевым условиям, а в точках $\xi_{i}$ - некоторым условиям согласования, связывающим между собой значения функции и односторонних производных справа и слева от точки разрыва. 
2. Идеи и методы. В основе обоснования знакорегулярных свойств краевых задач лежат три ключевых принципа (см. [4], [7]-[10], [13], [17], [18]). Первьй - это использование дифференциального оператора, приведенного к факторизованной форме Фробениуса-Пойа [19],

$$
L y=h_{n}(x) \frac{d}{d x} h_{n-1}(x) \frac{d}{d x} \ldots h_{1}(x) \frac{d}{d x}\left[h_{0}(x) y\right],
$$

где $h_{i}(x)$ - достаточно (для того, чтобы дифференцирование в (5) было классическим) гладкие строго положительные на $[a, b]$ функции ${ }^{1}$. Использование этой формы отвечает существу дела: для знакорегулярности двухточечной задачи на отрезке $[a, b]$ необходимо, чтобы оператор был представим в виде (5) как минимум на полуинтервале $[a, b)$ (см. [4]). Поэтому обычно предполагается, что оператор уже задан в виде (5) (формулы преобразования к виду (5), выражающие $h_{i}(x)$ через вронскианы фундаментальной системы решений, можно найти в [19]).

Форма (5) позволяет применять при исследовании знаковых свойств технику теоремы Ролля (из того, что $u^{\prime}(x)$ меняет знак $k$ раз следует, что $u(x)$ меняет знак не более $k+1$ раз - не более одного раза на каждом из $(k+1)$ промежутков знакопостоянства производной), дающей “элементарное” неравенство $S(u) \leqslant S\left(u^{\prime}\right)+1$. Последовательное использование в этом неравенстве в качестве $u(x)$ каждой из квазипроизводных

$$
D_{0} y=h_{0}(x) y, \quad D_{i+1} y=h_{i+1}(x) \frac{d}{d x} D_{i} y
$$

позволяет приходить, для операторов $L$ вида (5), к неравенствам типа

$$
S(y) \leqslant S(L y)+n .
$$

Второй принцип - использование, для уточнения неравенства (7), идей, лежащих в основе правила Декарта [22, отдел V]. Правило Декарта, в котором число нулей полинома $P_{n}(x)$ оценивается по разности числа перемен знака в наборе чисел $P_{n}(a), P_{n}^{\prime}(a), \ldots$, $P_{n}^{(n)}(a)$ и числа перемен знака в наборе чисел $P_{n}(b), P_{n}^{\prime}(b), \ldots, P_{n}^{(n)}(b)$, существенно улучшает "грубую" оценку числа нулей полинома $P_{n}(x)$ числом $n$ (которая, по существу, идентична $(7)$ - для $y(x)=P_{n}(x)$ при $\left.L y \equiv y^{(n)}\right)$. Для полиномов, имеющих только вещественные нули, оценка из правила Декарта точна, так что она является достаточно тонким инструментом, и использование аналогичных соображений позволяет за счет знания числа перемен знака в наборах граничных значений $D_{i} y(a)$ и $D_{i} y(b)$ уменьшить правую часть в (7).

Оценку же числа перемен знака в наборах граничных значений $D_{i} y(a)$ и $D_{i} y(b)$ удается сделать, пользуясь одним эффективно проверяемым свойством матриц коэффициентов краевых условий (и условий согласования - в случае разрьвной задачи). Здесь ключевую роль играет одна теорема из [6], которую мы процитируем чуть ниже.

Отметим, что основные сложности в обосновании знакорегулярности по указанной схеме порождаются тем фактом, что квазипроизводные решения могут, вообще говоря, обращаться в нуль на концах отрезка, и это создает некоторьй “зазор” в трактовке

\footnotetext{
${ }^{1}$ Оператор допускает такое представление тогда и только тогда, когда фундаментальная система решений уравнения $L y=0$ является системой Чебышева. Связь между возможностью факторизации и свойствами фундаментальной системы решений является предметом теории, в отечественной литературе назьваемой теорией неосиилляиии [20], а в иностранной - disconjugacy theory [21].
} 
понятия числа перемен знака в наборах граничных значений $D_{i} y(a)$ и $D_{i} y(b)$; для уточнения оценок требуется перебор многочисленных вариантов и подвариантов, так что в конще концов рассуждения “тонут" в этом лабиринте. Основным техническим достижением настоящей работы является удачньй выбор обобщения правила Декарта, которое, с одной стороны, точно “вписьвается" в упомянутую вьше теорему из [6], а с другойне требует при использовании его в анализе краевой задачи никаких дополнительных оговорок.

Эффективность этого обобщения позволила не только сделать совершенно прозрачными и ясными уже известные условия знакорегулярности для двухточечных задач условия Калафати [23] (см. также [7]), но и обосновать их аналог для существенно более сложной разрывной краевой задачи.

3. Определения и формулировки. Пусть $\Omega=(a, b) \backslash\left\{\xi_{i}\right\}_{i=1}^{r}$, где $a<\xi_{1}<$ $\xi_{2}<\cdots<\xi_{r}<b$. Для единообразия будем считать $a=\xi_{0}, b=\xi_{r+1}$. Через $C[\Omega]$ обозначим пространство кусочно-непрерывных функций, допускающих разрьвы только первого рода и только в точках $\xi_{i}$; для функции $u(x) \in C[\Omega]$ под $u\left(\xi_{i} \pm 0\right)$ будем понимать соответствующие пределы. Через $C^{n}[\Omega]$ обозначим пространство функций из $C[\Omega]$, имеющих $n$ производных, также принадлежащих $C[\Omega]$. Можно формально считать, что $C^{n}[\Omega]=\prod_{i=0}^{r} C^{n}\left[\xi_{i}, \xi_{i+1}\right]$.

Пусть $L$ - дифференциальный оператор порядка $n$ вида $(5), D_{j} y-$ квазипроизводные (6). Предполагая уравнение

$$
L y=f(x)
$$

заданным на $\Omega$ (т.е. на $[a, b]$ без точек $\xi_{i}$ ), снабдим его в точках $a$ и $b$ краевыми условиями, приведенными к виду

$$
\begin{aligned}
\sum_{j=1}^{n} \alpha_{k j}(-1)^{j-1} D_{j-1} y(a) & =0, \quad k=1, \ldots, p \\
\sum_{j=1}^{n} \beta_{k j} D_{j-1} y(b) & =0, \quad k=1, \ldots, q
\end{aligned}
$$

$(p+q=n)$, а в каждой точке разрьва $\xi_{i}-$ условиями согласования, приведенными к виду

$$
\sum_{j=1}^{n} \alpha_{k j}^{i,-} D_{j-1} y\left(\xi_{i}-0\right)-\sum_{j=1}^{n} \alpha_{k j}^{i,+}(-1)^{j-1} D_{j-1} y\left(\xi_{i}+0\right)=0, \quad k=1, \ldots, n
$$

$(i=1, \ldots, r-$ номер точки, в которой задается условие; $k$ - номер условия в серии условий, заданных в этой точке - в каждой серии предполагается заданными точно $n$ условий; $j$ - индекс суммирования и одновременно номер производной, на которую умножается соответствующий коэффициент).

Задачу (8)-(10) мы и будем в дальнейшем назьвать разрывной краевой задачей.

Для описания свойства знакорегулярности введем понятие числа перемен знака функиии. Это понятие, интуитивно ясное, например, для полиномов, в более тонких ситуациях (когда функция имеет неизолированные нули) требует уточнения в рафинированных терминах. 
Интервал $\left(a_{1}, a_{2}\right) \subset[a, b]$ назьвается промежутком знакопостоянства функции, если на этом интервале функция сохраняет знак и не тождественна нулю.

Говорят, что функция $u(x) \in C[\Omega]$ имеет $k$ перемен знака и пишут $S(u)=k$, если $[a, b]$ может быть разбит на $k+1$ промежуток знакопостоянства функции так, что на соседних промежутках $u(x)$ имеет разный знак.

Если $u(x)$ имеет конечное число изолированных нулей, то точками единственно возможного разбиения являются те нули, в окрестности которых функция меняет знак (что как раз соответствует интуитивному представлению). Если же функция имеет сплошные нули, то разбиение можно выбирать разными способами, но число перемен знака от этого выбора не зависит. Для $u(x)$, знакопостоянной на всем $[a, b]$, полагается $S(u)=0$; для $u(x) \equiv 0$ обычно считается $S(u)=-1$ (оно позволяет распространить “элементарное" неравенство $S(u) \leqslant S\left(u^{\prime}\right)+1$ и на случай $u^{\prime} \equiv 0$, т.е. $u \equiv$ const). Для тех же $u(x)$, для которых не существует разбиения $[a, b]$ на конечное число промежутков знакопостоянства, считается $S(u)=+\infty$.

ОПРЕДЕЛЕНИЕ. Задачу (8)-(10) будем назьвать знакорегулярной, если она однозначно разрешима и если для любой $f(x)$ и соответствуюшего решения $y(x)$ вьполнено $S(y) \leqslant S(f)$.

Конечно, содержательньм это свойство является только по отношению к тем $f(x)$, для которых $S(f)$ конечно.

Для формулировки основного результата нам понадобится следующее свойство матриц, образованных коэффициентами краевых условий (оно играет ключевую роль для знакорегулярности краевой задачи).

ОПрЕДЕЛЕниЕ. Матрица $A$ размера $k \times n(k \leqslant n)$ называется знакосогласованной, если она имеет ранг $k$ и все ненулевые миноры ранга $k$ имеют один и тот же знак.

Основное значение свойства знакосогласованности состоит в том, что оно позволяет оценить число перемен знака у решения $y=\left(y_{1}, \ldots, y_{m}\right)$ линейной системы $A y=0$. Для того, чтобы сформулировать эту оценку (которая и является предметом упоминавшейся нами теоремы из [6]), нам понадобится уточнить понятие “число перемен знака" для последовательности чисел.

Пусть $y_{0}, y_{1}, \ldots, y_{m}$ - набор ненулевых чисел. Через $S\left(y_{0}, y_{1}, \ldots, y_{m}\right)$ обозначается число перемен знака в наборе $y_{0}, y_{1}, \ldots, y_{m}$. Формально $S(\cdot)$ определяется формулами

$$
S\left(y_{0}, y_{1}, \ldots, y_{m}\right)=S\left(y_{0}, y_{1}\right)+S\left(y_{1}, y_{2}\right)+\cdots+S\left(y_{m-1}, y_{m}\right)
$$

и

$$
S(y, z)=\left\{\begin{array}{l}
1, \text { если } y z<0, \\
0, \text { если } y z>0 .
\end{array}\right.
$$

Мы будем пользоваться, помимо $(11),(12)$, следующими очевидными свойствами $S(\cdot)$ :

$$
\begin{aligned}
S\left(y_{0}, y_{1}, \ldots, y_{m}\right)= & S\left(-y_{0},-y_{1}, \ldots,-y_{m}\right)=S\left(y_{m}, y_{m-1}, \ldots, y_{0}\right) \\
& S\left(y_{0}, y_{1}\right)+S\left(y_{0},-y_{1}\right)=1
\end{aligned}
$$

Пусть теперь $\left(y_{0}, y_{1}, \ldots, y_{m}\right)$ - набор чисел, среди которых есть и нулевые. Для такого набора появляется возможность варьировать характеристику “число перемен знака", поскольку нулям можно приписывать как положительньй, так и отрицательньй 
знак: так, например, возможны варианты $S(1,-2,+0,-0,1)=4$ или $S(1,-2,-0$, $-0,1)=2$. Максимальное из получаемых таким образом чисел мы обозначим, следуя [6], через $S^{+}$, а минимальное- через $S^{-}$. Они реализуются: $S^{+}$- когда нулю приписывается знак, противоположньй знаку соседнего (например, левого) элемента в набоpe, a $S^{-}$- когда знак нуля, наоборот, считается таким же, как знак соседнего элемента. В нашем примере как раз $S^{+}(1,-2,0,0,1)=4, S^{-}(1,-2,0,0,1)=2$.

Теорема 1 [6, гл. $5, \S 2$, теорема 7]. Для того, чтобы для любого ненулевого решения $y=\left(y_{1}, y_{2}, \ldots, y_{m}\right)$ системы $A y=0 c(k \times m)$-матрицей $A(k \leqslant m)$ было выполнено $S^{+}\left(y_{1}, y_{2}, \ldots, y_{m}\right) \geqslant k$, необходимо и достаточно, чтобы матрииа $A$ была знакосогласована.

Теперь мы можем сформулировать основной результат данной работы.

Теорема 2. Пусть А и В - матрииы коэффициентов краевых условий (9)

$$
A=\left\|\alpha_{k j}\right\|_{k=1, \ldots, p}^{j=1, \ldots, n}, \quad B=\left\|\beta_{k j}\right\|_{k=1, \ldots, q}^{j=1, \ldots, n},
$$

$A_{i}^{ \pm}$-матрицы, образованные коэффициентами условий согласования (10),

$$
A_{i}^{+}=\left\|\alpha_{k j}^{i,+}\right\|_{k=1, \ldots, n}^{j=1, \ldots, n}, \quad A_{i}^{-}=\left\|\alpha_{k j}^{i,-}\right\|_{k=1, \ldots, n}^{j=1, \ldots, n},
$$

а $A_{i}-(n \times 2 n)$-матрицы, образованные столбиами $A_{i}^{-}$, идущими в обратном порядке, а затем столбиами $A_{i}^{+}$, идущими в прямом порядке.

Задача (8)-(10) является знакорегулярной, если она однозначно разрешима и каждая из матрии $A, B, A_{i}$ является знакосогласованной.

В случае, когда точки разрьва $\xi_{i}$ отсутствуют, наша теорема идентична теореме Калафати [23], [7].

4. Обобщенное правило Декарта. Пусть $y \in C[\Omega]$ и имеет конечное число нулей на $[a, b]$. Через $\sigma y(x \pm 0)$ обозначим знак $y(x)$ справа (слева) от точки $x$ (в формулах, где это необходимо, мы будем знак \pm отождествлять с числом \pm 1$)$. Если $x \neq \xi_{i}$ и $y(x) \neq 0$, то, очевидно, $\sigma y(x+0)=\sigma y(x-0)=\operatorname{sign} y(x)$. Если же $x \in\left\{\xi_{i}\right\}_{i=1}^{r}$, то $\sigma y(x+0)$ и $\sigma y(x-0)$, вообще говоря, различны и совпадают со знаками значений $y(x+0)$ и $y(x-0)$, если последние не равны нулю. Использование $\sigma y(x \pm 0)$ позволяет нам в случаях, когда $y(x \pm 0)=0$, приписьвать этому нулю вполне определенный знак.

ЛЕмма 1 (обобщенная теорема Ролля). Пусть $u(x) \in C^{1}[a, b] u u^{\prime}(x)$ uмеeт конечное число нулей. Тогда

$$
S(u) \leqslant S\left(u^{\prime}\right)+1-S\left(\sigma u(a+0),-\sigma u^{\prime}(a+0)\right)-S\left(\sigma u(b-0), \sigma u^{\prime}(b-0)\right) .
$$

ДоКАЗАТЕльСтво по существу сводится к эквиваленту теоремы Ролля: на промежутке, где производная сохраняет знак, функция монотонна и меняет знак не более одного раза. Поэтому в условиях леммы число перемен знака $u(x)$ не превосходит числа промежутков знакопостоянства производной (т.е. $\left.S\left(u^{\prime}\right)+1\right)$. Если при этом справа от точки $a$ знаки $u(x)$ и $u^{\prime}(x)$ совпадают (что соответствует $\left.S\left(\sigma u(a+0),-\sigma u^{\prime}(a+0)\right)=1\right)$, то на первом промежутке знакопостоянства производной сама функция $u(x)$ знака не меняет. Аналогично последнее слагаемое в (15) учитывает возможность отсутствия перемены знака у функции $u(x)$ на последнем промежутке знакопостоянства производной при совпадении $\sigma u(b-0)$ и $\left(-\sigma u^{\prime}(b-0)\right)$. 
ЗАмЕчАниЕ. Утверждение леммы 1 сохраняет силу при замене обычной производной на квазипроизводную $D u \equiv h(x) u^{\prime}$, где $h(x)$ - непрерывная строго положительная функция.

ЛЕмма 2 (обобщенное правило Декарта). Пусть $y(x) \in C^{n}[a, b]$ u $y^{(n)}(x)$ uмеет конечное число нулей. Тогда

$$
\begin{gathered}
S(y) \leqslant S\left(y^{(n)}\right)+n-S\left(\sigma y(a+0),-\sigma y^{\prime}(a+0), \ldots,(-1)^{n} \sigma y^{(n)}(a+0)\right) \\
-S\left(\sigma y(b-0), \sigma y^{\prime}(b-0), \ldots, \sigma y^{(n)}(b-0)\right) .
\end{gathered}
$$

ДоКАЗАТЕЛЬСТВО сводится к сложению формул (15), записанных для $u(x)=y^{j}(x)$ для $j$ от нуля до $n-1$, и приведением, используя (11) и (13), сумм $S\left(\sigma y(a+0),-\sigma y^{\prime}(a+0)\right)+S\left(\sigma y^{\prime}(a+0),-\sigma y^{\prime \prime}(a+0)\right)+\cdots+S\left(\sigma y^{(n-1)}(a+0),-\sigma y^{(n)}(a+0)\right)$ и $S\left(\sigma y(b-0), \sigma y^{\prime}(b-0)\right)+S\left(\sigma y^{\prime}(b-0), \sigma y^{\prime \prime}(b-0)\right)+\cdots+S\left(\sigma y^{(n-1)}(b-0), \sigma y^{(n)}(b-0)\right)$ к соответствующим слагаемым в (16).

Классическое правило Декарта получается из леммы 2 при $y^{(n)} \equiv 1:$ в этом случае $S\left(y^{(n)}\right)=0, \mathrm{a}$

$$
\begin{gathered}
n-S\left(\sigma y(a+0),-\sigma y^{\prime}(a+0), \ldots,(-1)^{n} \sigma y^{(n)}(a+0)\right) \\
=S\left(\sigma y(a+0), \sigma y^{\prime}(a+0), \ldots, \sigma y^{(n)}(a+0)\right) .
\end{gathered}
$$

Заменой в рассуждениях обычных производных на квазипроизводные $D_{i} y$, определяемые (6), получается

ЛЕмма $2^{\prime}$. Пусть $L-\partial и ф ф е р е н и и а л ь н ы и ̆ ~ о п е р а т о р ~ в и д а ~(5), ~ y(x) \in C^{n}[a, b] u$ $L y(x) \equiv D_{n} y(x)$ имеет конечное число нулей. Тогда

$$
\begin{gathered}
S(y) \leqslant S(L y)+n-S\left(\sigma y(a+0),-\sigma D_{1} y(a+0), \ldots,(-1)^{n} \sigma D_{n} y(a+0)\right) \\
-S\left(\sigma y(b-0), \sigma D_{1} y(b-0), \ldots, \sigma D_{n} y(b-0)\right) .
\end{gathered}
$$

5. Теорема Калафати. Как следует из формулы $\left(16^{\prime}\right)$, знакорегулярность задачи (8)-(10) в случае, когда точки разрыва $\xi_{i}$ отсутствуют, наверняка обеспечивается неравенством

$$
\begin{aligned}
& S\left(\sigma y(a+0),-\sigma D_{1} y(a+0), \ldots,(-1)^{n} \sigma D_{n} y(a+0)\right) \\
& \quad+S\left(\sigma y(b-0), \sigma D_{1} y(b-0), \ldots, \sigma D_{n} y(b-0)\right) \geqslant n .
\end{aligned}
$$

Знакосогласованность матриц $A$ и $B$ позволяет в силу теоремы 1 немедленно получить неравенства

$$
\begin{aligned}
& S^{+}\left(y(a+0),-D_{1} y(a+0), \ldots,(-1)^{n} D_{n} y(a+0)\right) \\
& \quad \geqslant S^{+}\left(y(a+0),-D_{1} y(a+0), \ldots,(-1)^{n-1} D_{n-1} y(a+0)\right) \geqslant p(=\operatorname{rang} A), \\
& S^{+}\left(y(b-0), D_{1} y(b-0), \ldots, D_{n} y(b-0)\right) \\
& \quad \geqslant S^{+}\left(y(b-0), D_{1} y(b-0), \ldots, D_{n} y(b-0)\right) \geqslant q(=\operatorname{rang} B)
\end{aligned}
$$


(напомним, что $p+q=n$ ).

Удача же оказывается в том, что для всех $y(x) \in C^{n}[a, b]$, для которых $D_{n} y(a)$ и $D_{n} y(b)$ отличны от нуля,

$$
S\left(\sigma y(a+0),-D_{1} \sigma y(a+0), \ldots,(-1)^{n} \sigma D_{n} y(a+0)\right)
$$

в точности совпадает с

$$
S^{+}\left(y(a+0),-D_{1} y(a+0), \ldots,(-1)^{n} D_{n} y(a+0)\right)
$$

(и аналогично - в точке $b$ ). Действительно, если все $D_{j} y(a) \neq 0$, то и $(20)$, и $\left(20^{\prime}\right)$ - это просто число перемен знака в наборе $\left(y(a),-D_{1} y(a), \ldots,(-1)^{n} D_{n} y(a)\right)$. Если же среди $D_{j} y(a)$ есть нулевые, то для любой последовательности нулей в наборе производных

$$
D_{k} y(a)=\cdots=D_{l-1} y(a)=0, \quad D_{l} y(a) \neq 0
$$

знаки $\sigma D_{j} y(a+0)$ всех квазипроизводных $D_{j} y(j=k, \ldots, l)$ будут, очевидно, совпадать со знаком $D_{l} y(a)$. Поэтому соответствующая часть

$$
\ldots(-1)^{k} \sigma D_{k} y(a+0), \ldots,(-1)^{l} \sigma D_{l} y(a+0) \ldots
$$

набора в (20) будет состоять из чередующихся плюс-минус единиц, а это как раз соответствует определению $S^{+}$в $\left(20^{\prime}\right)$.

Доказательство теоремы Калафати (теоремы 2 для случая, когда точки разрыва отсутствуют) теперь оказывается совсем простым. Для $f \in C[a, b]$, имеющей конечное число нулей и не обращающейся в нуль в точках $a$ и $b$, неравенство $S(y) \leqslant S(f) \quad(=$ $\left.S\left(D_{n} y\right)\right)$ следует из $\left(16^{\prime}\right),(17)$, где $(17)$ обосновьвается знакосогласованностью матрищ краевых условий (формулы (18), (19) с учетом совпадения $(20)$ и $\left(20^{\prime}\right)$ ).

Для всех же остальньх $f(x) \in C[a, b]$ знакорегулярность обосновывается методом возмущений: к $f(x)$ добавляется многочлен $\epsilon P(x)$, где $P(x)$ выбирается меняющим знак синхронно с $f(x)$ и не обращающимся в нуль в точках $a$ и $b$. В этом случае $S(f+\epsilon P)=$ $S(f)$; для соответствующих решений $y_{\epsilon}$ получаем $S\left(y_{\epsilon}\right) \leqslant S(f+\epsilon P)=S(f)$, и предельный переход дает нам неравенство $S(y) \leqslant S(f)$ благодаря полунепрерывности снизу функционала $S(y)$ (см. [7]): из $y_{\epsilon}(x) \rightarrow y(x)$ поточечно на $[a, b]$ следует

$$
S(y) \leqslant \liminf _{\epsilon \rightarrow 0} S\left(y_{\epsilon}\right)
$$

6. Правило Декарта с разрывами. Для доказательства теоремы 2 в случае наличия точек разрьва $\xi_{i}$ нам, естественно, понадобятся аналоги лемм 1 и 2 , учитьвающие возможность разрывов в точках $\xi_{i}$. Чтобы сфформулировать эти аналоги, нам понадобится ряд дополнительных понятий.

Введем в рассмотрение ииклическое и антиииклическое число перемен знака в наборе $\left(y_{0}, y_{1}, \ldots, y_{m}\right)$, обозначая их через $S_{\text {。 }}\left(y_{0}, y_{1}, \ldots, y_{m}\right)$ и $S_{\mu}\left(y_{0}, y_{1}, \ldots, y_{m}\right)$ соответственно и определяя как

$$
\begin{aligned}
& S_{\circ}\left(y_{0}, y_{1}, \ldots, y_{m}\right)=S\left(y_{0}, y_{1}\right)+S\left(y_{1}, y_{2}\right)+\cdots+S\left(y_{m-1}, y_{m}\right)+S\left(y_{m}, y_{0}\right) \\
& S_{\mu}\left(y_{0}, y_{1}, \ldots, y_{m}\right)=S\left(y_{0}, y_{1}\right)+S\left(y_{1}, y_{2}\right)+\cdots+S\left(y_{m-1}, y_{m}\right)+S\left(y_{m},-y_{0}\right)
\end{aligned}
$$

Названия оправдываются тем, что

$$
\begin{aligned}
& S_{\circ}\left(y_{0}, y_{1}, \ldots, y_{m}\right)=S_{\circ}\left(y_{m}, y_{0}, y_{1}, \ldots, y_{m-1}\right), \\
& S_{\mu}\left(y_{0}, y_{1}, \ldots, y_{m}\right)=S_{\mu}\left(-y_{m}, y_{0}, y_{1}, \ldots, y_{m-1}\right) .
\end{aligned}
$$

Эти формулы означают, что элементы в наборе можно переставлять “по кругу" с сохранением $S_{\text {。 }}\left(S_{\mu}\right)$, изменяя при этом, в случае $S_{\mu}$, знак у переставляемого элемента. 
Лемма 3. Пусть $u(x) \in C^{1}[\Omega]$ и $u^{\prime}(x)$ имеет конечное число нулей на $\Omega$. Тогда

$$
\begin{gathered}
S(u) \leqslant S\left(u^{\prime}\right)+2 r+1-S\left(\sigma u(a+0),-\sigma u^{\prime}(a+0)\right)-S\left(\sigma u(b-0), \sigma u^{\prime}(b-0)\right) \\
-\sum_{i=1}^{r} S_{\circ}\left(\sigma u^{\prime}\left(\xi_{i}-0\right), \sigma u\left(\xi_{i}-0\right),-\sigma u\left(\xi_{i}+0\right), \sigma u^{\prime}\left(\xi_{i}+0\right)\right) .
\end{gathered}
$$

ДокАЗАТЕЛЬСТво. Сложением формул (15), записанных для каждого $\left[\xi_{i}, \xi_{i+1}\right]$, с учетом

$$
S\left(\left.v\right|_{[a, b]}\right)=\sum_{i=0}^{r} S\left(\left.v\right|_{\left[\xi_{i}, \xi_{i+1}\right]}\right)+\sum_{i=1}^{r} S\left(\sigma v\left(\xi_{i}-0\right), \sigma v\left(\xi_{i}+0\right)\right)
$$

(для $v(x)=u(x)$ и $v(x)=u^{\prime}(x)$ ), получаем

$$
\begin{aligned}
S(u) \leqslant & S\left(u^{\prime}\right)+r+1-S\left(\sigma u(a+0),-\sigma u^{\prime}(a+0)\right)-S\left(\sigma u(b-0), \sigma u^{\prime}(b-0)\right) \\
& -\sum_{i=1}^{r} S\left(\sigma u^{\prime}\left(\xi_{i}-0\right), \sigma u\left(\xi_{i}-0\right)\right)-\sum_{i=1}^{r} S\left(-\sigma u^{\prime}\left(\xi_{i}+0\right), \sigma u\left(\xi_{i}+0\right)\right) \\
& -\sum_{i=1}^{r} S\left(\sigma u^{\prime}\left(\xi_{i}+0\right), \sigma u^{\prime}\left(\xi_{i}-0\right)\right)+\sum_{i=1}^{r} S\left(\sigma u\left(\xi_{i}-0\right), \sigma u\left(\xi_{i}+0\right)\right) .
\end{aligned}
$$

Оказалось естественным заменить каждую

$$
S\left(\sigma u\left(\xi_{i}-0\right), \sigma u\left(\xi_{i}+0\right)\right)
$$

на

$$
1-S\left(\sigma u\left(\xi_{i}-0\right),-\sigma u\left(\xi_{i}+0\right)\right)
$$

(формула (14)) и, воспользовавшись свойством

$$
S\left(\sigma u\left(\xi_{i}+0\right),-\sigma u^{\prime}\left(\xi_{i}+0\right)\right)=S\left(-\sigma u\left(\xi_{i}+0\right), \sigma u^{\prime}\left(\xi_{i}+0\right)\right)
$$

(формула (13)), объединить

$$
\begin{aligned}
& \sum_{i=1}^{r} S\left(\sigma u^{\prime}\left(\xi_{i}-0\right), \sigma u\left(\xi_{i}-0\right)\right)+\sum_{i=1}^{r} S\left(\sigma u^{\prime}\left(\xi_{i}+0\right),-\sigma u\left(\xi_{i}+0\right)\right) \\
& \quad+\sum_{i=1}^{r} S\left(\sigma u^{\prime}\left(\xi_{i}+0\right), \sigma u^{\prime}\left(\xi_{i}-0\right)\right)+\sum_{i=1}^{r} S\left(\sigma u\left(\xi_{i}-0\right),-\sigma u\left(\xi_{i}+0\right)\right)
\end{aligned}
$$

B

$$
S_{\circ}\left(\sigma u^{\prime}\left(\xi_{i}-0\right), \sigma u\left(\xi_{i}-0\right),-\sigma u\left(\xi_{i}+0\right), \sigma u^{\prime}\left(\xi_{i}+0\right)\right),
$$

получив тем самым из (24) формулу (23). Лемма доказана.

Как нетрудно понять, далее нам придется суммировать формулы (23) для всех производных функции $y(x) \in C^{n}[\Omega]$, поэтому отметим следующее правило суммирования $S_{\text {。 }}(\cdot)$ :

$$
\begin{aligned}
& S_{\circ}\left(y_{1}, y_{0},-z_{0}, z_{1}\right)+S_{\circ}\left(y_{2}, y_{1},-z_{1}, z_{2}\right)+\cdots+S_{\circ}\left(y_{m}, y_{m-1},-z_{m-1}, z_{m}\right) \\
& =m-1+ \begin{cases}S_{\circ}\left(y_{m}, y_{m-1}, \ldots, y_{0},-z_{0}, z_{1}, \ldots,(-1)^{m} z_{m-1},(-1)^{m+1} z_{m}\right), & m=2 l+1, \\
S_{\mu}\left(y_{m}, y_{m-1}, \ldots, y_{0},-z_{0}, z_{1}, \ldots,(-1)^{m} z_{m-1},(-1)^{m+1} z_{m}\right), & m=2 l .\end{cases}
\end{aligned}
$$


Формула (25) проверяется непосредственно - разложением каждого слагаемого на “атомы” по формулам $(21),(22)$, заменой каждой пары $S\left(y_{i}, z_{i}\right)+S\left(y_{i},-z_{i}\right)$ на 1 и последующим объединением оставшихся слагаемых в $S_{\circ}\left(S_{\mu}\right)$. Нам представляется более наглядным описать рекуррентный процесс с помощью формул

$$
\begin{gathered}
S_{\circ}\left(y_{k}, y_{k-1}, \ldots, y_{0},-z_{0}, z_{1}, \ldots,(-1)^{k} z_{k-1},(-1)^{k+1} z_{k}\right)+S_{\circ}\left(y_{k+1}, y_{k},-z_{k}, z_{k+1}\right) \\
\quad=1+S_{\mu}\left(y_{k+1}, y_{k}, \ldots, y_{0},-z_{0}, z_{1}, \ldots,(-1)^{k+1} z_{k},(-1)^{k+2} z_{k+1}\right), \\
S_{\mu}\left(y_{k}, y_{k-1}, \ldots, y_{0},-z_{0}, z_{1}, \ldots,(-1)^{k} z_{k-1},(-1)^{k+1} z_{k}\right)+S_{\circ}\left(y_{k+1}, y_{k},-z_{k}, z_{k+1}\right) \\
=1+S_{\circ}\left(y_{k+1}, y_{k}, \ldots, y_{0},-z_{0}, z_{1}, \ldots,(-1)^{k+1} z_{k},(-1)^{k+2} z_{k+1}\right) .
\end{gathered}
$$

Доказательство этих формул также основьвается на разложении и использовании (14), но сами формулы определяют закон “нарашивания" количества элементов $y_{j}, z_{j}$ в наборе; при таком "наращивании” антициклическое число перемен знака, оказьвается, играет ту же роль, что и циклическое: они переходят друг в друга при "подклеивании" к ним “элементарного” цикла.

Лемма 4. Пусть $y(x) \in C^{n}[\Omega]$ и $y^{(n)}(x)$ имеет конечное число нулей. Тогда

$$
\begin{aligned}
S(y) \leqslant & S\left(y^{(n)}\right)+n+r(n+1)-S\left(\sigma y(a+0),-\sigma y^{\prime}(a+0), \ldots,(-1)^{n} \sigma y^{(n)}\right)(a+0) \\
& -S\left(\sigma y(b-0), \sigma y^{\prime}(b-0), \ldots, \sigma y^{(n)}\right)(b-0) \\
& -\sum_{i=1}^{r} S_{\circ \mu}\left(\sigma y^{(n)}\left(\xi_{i}-0\right), \ldots, \sigma y\left(\xi_{i}-0\right),-\sigma y\left(\xi_{i}+0\right), \ldots,(-1)^{n+1} \sigma y^{(n)}\left(\xi_{i}+0\right)\right)
\end{aligned}
$$

(в последнем слагаемом через $S_{\circ \mu}$ обозначено $S_{\circ} n p u n=2 l+1 u S_{\mu}-n p u n=2 l$ ).

ДокАЗАТЕльство получается сложением формул $(23)$ для $u(x)=y^{(j)}(x)(\mathrm{c} j=$ $0, \ldots, n-1)$ ) и использованием $(25)$ (при этом слагаемое $2 r \cdot n$ уменьшается в силу $(25)$ на величину $r(n-1)$ и от него остается $r(n+1))$.

Так же, как и в случае лемм 1,2 , утверждения лемм 3,4 остаются полностью справедливыми при замене обычных производных функции $y(x)$ на квазипроизводные $D_{i} y(x)$, определяемые формулами (6). Так получается

Лемма $4^{\prime}$. Пусть $L-$-ифференциальный оператор вида (5) на $\Omega, y(x) \in C^{n}[\Omega]$ и Ly $(x)$ имеет конечное число нулей. Тогда

$$
\begin{aligned}
S(y) \leqslant & S(L y)+n+r(n+1)-S\left(\sigma y(a+0),-\sigma D_{1} y(a+0), \ldots,(-1)^{n} \sigma D_{n} y(a+0)\right) \\
& -S\left(\sigma y(b-0), \sigma D_{1} y(b-0), \ldots, \sigma D_{n} y(b-0)\right) \\
& -\sum_{i=1}^{r} S_{\circ \mu}\left(\sigma D_{n} y\left(\xi_{i}-0\right), \ldots, \sigma y\left(\xi_{i}-0\right),-\sigma y\left(\xi_{i}+0\right), \ldots,(-1)^{n+1} \sigma D_{n} y\left(\xi_{i}+0\right)\right) .
\end{aligned}
$$


7. Доказательство теоремы 2 в случае наличия точек разрыва $\xi_{i}$. Рассмотрим сначала случай, когда $f\left(=L y \equiv D_{n} y\right) \in C[\Omega]$ имеет конечное число нулей и не обращается в нуль в точках $\xi_{i} \pm 0$. Тогда в силу знакосогласованности матриц $A$ и $B$ дословньм повторением рассуждений п. 5 получаем в силу теоремы 1

$$
\begin{aligned}
& S\left(\sigma y(a+0),-\sigma D_{1} y(a+0), \ldots,(-1)^{n} \sigma D_{n} y(a+0)\right) \\
& \quad+S\left(\sigma y(b-0), \sigma D_{1} y(b-0), \ldots, \sigma D_{n} y(b-0)\right) \geqslant p+q=n .
\end{aligned}
$$

Кроме того, из знакосогласованности каждой из матриц $A_{i}$ следует опять же в силу теоремы 1, что

$$
\begin{aligned}
S\left(\sigma D_{n} y\left(\xi_{i}-0\right), \ldots, \sigma y\left(\xi_{i}-0\right),-\sigma y\left(\xi_{i}+0\right), \ldots,(-1)^{n+1} \sigma D_{n} y\left(\xi_{i}+0\right)\right) & \\
= & S^{+}\left(D_{n} y\left(\xi_{i}-0\right), \ldots, y\left(\xi_{i}-0\right),-y\left(\xi_{i}+0\right), \ldots,(-1)^{n+1} D_{n} y\left(\xi_{i}+0\right)\right) \\
& \geqslant S^{+}\left(D_{n-1} y\left(\xi_{i}-0\right), \ldots, y\left(\xi_{i}-0\right),-y\left(\xi_{i}+0\right), \ldots,(-1)^{n} D_{n-1} y\left(\xi_{i}+0\right)\right) \geqslant n,
\end{aligned}
$$

так как фигурирующий в последней строке вектор как раз является решением системы с матрицей $A_{i}$. Покажем, что из $(28)$ следует

$$
S_{\circ \mu}\left(\sigma D_{n} y\left(\xi_{i}-0\right), \ldots, \sigma y\left(\xi_{i}-0\right),-\sigma y\left(\xi_{i}+0\right), \ldots,(-1)^{n+1} \sigma D_{n} y\left(\xi_{i}+0\right)\right) \geqslant n+1
$$

Пусть, например, $n=2 l$ (для $n=2 l+1$ доказательство аналогичное). Тогда

$$
\begin{aligned}
& S_{\mu}\left(\sigma D_{n} y\left(\xi_{i}-0\right), \ldots, \sigma y\left(\xi_{i}-0\right),-\sigma y\left(\xi_{i}+0\right), \ldots,(-1)^{n+1} \sigma D_{n} y\left(\xi_{i}+0\right)\right) \\
&= S\left(\sigma D_{n} y\left(\xi_{i}-0\right), \ldots, \sigma y\left(\xi_{i}-0\right),-\sigma y\left(\xi_{i}+0\right), \ldots,(-1)^{n+1} \sigma D_{n} y\left(\xi_{i}+0\right)\right) \\
&+S\left(\sigma D_{n} y\left(\xi_{i}+0\right), \sigma D_{n} y\left(\xi_{i}-0\right)\right)
\end{aligned}
$$

(в последнем слагаемом $(-1)^{n+1}$ заменена в силу $n=2 p$ на $(-1)$ и общий минус в силу (13) опущен). Первое слагаемое в правой части (30) не меньше $n$ в силу (28). Если неравенство строгое, то уже это слагаемое не меньше $n+1$ и $(29)$ вьполнено. Если же первое слагаемое в правой части (30) в точности равно $n$, то первьй и последний элементы в соответствующем наборе (это $\sigma D_{n} y\left(\xi_{i}-0\right)$ и $(-1)^{n+1} \sigma D_{n} y\left(\xi_{i}+0\right)=-\sigma D_{n} y\left(\xi_{i}+0\right)$ ) имеют одинаковьй знак ( $n$ четно). Но тогда уже последнее слагаемое оказывается ненулевым:

$$
S\left(\sigma D_{n} y\left(\xi_{i}+0\right), \sigma D_{n} y\left(\xi_{i}-0\right)\right)=1,
$$

и неравенство (29) опять вьполнено.

Итак, знакосогласованность $A_{i}, A$ и $B$ обеспечивают неравенства (27) и (29), учет которых в $\left(28^{\prime}\right)$ дает

$$
S(y) \leqslant S(L y)=S(f) .
$$

Таким образом, обосновано утверждение теоремы 2 в случае, когда $f(x)$ имеет конечное число нулей и не обращается в нуль в точках $\xi_{i} \pm 0$.

В случае же произвольной $f(x)$, имеющей конечное число перемен знака, утверждение теоремы 2 обосновьвается использованием, как и в п. 5 , возмущений $f_{\epsilon}(x)=f(x)+$ $\epsilon P(x)$, где $P(x)$ - кусочно-полиномиальная функция, меняющая знак синхронно с $f(x)$ и не обращающаяся в нуль в точках $\xi_{i} \pm 0$. Этим замечанием завершается доказательство теоремы 2. 
8. Естественные обобщения. Предъявленная техника обоснования знакорегулярности допускает перенос на более сложные ситуации. Так, вполне понятно ее использование в случае, когда число условий согласования в каждой точке не точно совпадает с порядком уравнения, а меняется от точки к точке. Здесь для различных классов задач можно получать аналоги теоремы 2 , обосновьвая, с использованием знакосогласованности матрищ краевых условий, “итоговое” неравенство

$$
\begin{aligned}
& S\left(\sigma y(a+0),-\sigma y^{\prime}(a+0), \ldots,(-1)^{n} \sigma y^{(n)}\right)(a+0) \\
& \quad+S\left(\sigma y(b-0), \sigma y^{\prime}(b-0), \ldots, \sigma y^{(n)}\right)(b-0) \\
& \quad+\sum_{i=1}^{r} S_{\circ \mu}\left(\sigma y^{(n)}\left(\xi_{i}-0\right), \ldots, \sigma y\left(\xi_{i}-0\right),-\sigma y\left(\xi_{i}+0\right), \ldots,(-1)^{n+1} \sigma y^{(n)}\left(\xi_{i}+0\right)\right) \\
& \quad \geqslant n+r(n+1) .
\end{aligned}
$$

Более нетривиальным является распространение теоремы 2 на задачи с переменным порядком дифференциального оператора (на каждом $\left[\xi_{i}, \xi_{i+1}\right]$ свой). Здесь для обоснования соответствующего результата придется вместо схемы лемм 3,4 использовать другую: сначала просуммировать неравенства (15) для разных производных на каждом промежутке в отдельности, а затем уже "склеить" их в точках $\xi_{i}$.

Отметим, наконец, что рассуждения в доказательстве теоремы 2 (пп. 5,7 ) не изменяются, если расширить класс краевых условий, допуская в условиях (9), (10) суммирование по порядку производных не до $n-1$, а до $n$. Это соответствует постановке спектральных задач (1), в которых спектральньй параметр входит не только в правую часть дифференциального уравнения, но и в краевые условия и в условия согласования в виде слагаемых типа $\lambda \kappa_{k}^{i, \pm} y\left(\xi_{i} \pm 0\right)$, которые можно интерпретировать как

$\lambda \kappa_{k}^{i, \pm} y\left(\xi_{i} \pm 0\right)=\lambda q\left(\xi_{i} \pm 0\right) y\left(\xi_{i} \pm 0\right) \frac{\kappa_{k}^{i, \pm}}{q\left(\xi_{i} \pm 0\right)}=\frac{\kappa_{k}^{i, \pm}}{q\left(\xi_{i} \pm 0\right)}(L y)\left(\xi_{i} \pm 0\right)=\alpha_{k n}^{i, \pm}\left(D_{n} y\right)\left(\xi_{i} \pm 0\right)$

$c \alpha_{k n}^{i, \pm}=\kappa_{k}^{i, \pm} / q\left(\xi_{i} \pm 0\right)$. Такие задачи возникают, например, при изучении механических систем, содержащих как точечные, так и распределенные массы.

Автор искренне благодарит Ю. В. Покорного, открывшего ему богатство и красоту осцилляционной теории и А. А. Шкаликова за чрезвычайно полезные и интересные обсуждения.

\section{СПИСОК ЦИТИРОВАННОЙ ЛИТЕРАТУРЫ}

[1] Sturm C. Memorè sur les èquations differentielles linèaires du second ordre // J. Math. Pures Appl. 1836. V. 1. P. 373-444.

[2] Kellogg O. D. Orthogonal Function Sets Arising from Integral Equations // Amer. J. Math. 1918. № 40. P. 145-154.

[3] Kellogg O. D. Interpolation Properties of Orthogonal Sets of Solutions of Differential Equations // Amer. J. Math. 1918. № 40. P. 220-234.

[4] Крейн М. Г. О несимметрических осцилляционных функциях Грина обыкновенных дифференциальных операторов // Докл. АН СССР. 1939. Т. 25. № 8. С. 643-646.

[5] Крейн М. Г. Осцилляционные теоремы для обыкновенных дифференциальных операторов произвольного порядка // Докл. АН СССР. 1939. Т. 25. № 9. С. 717-720.

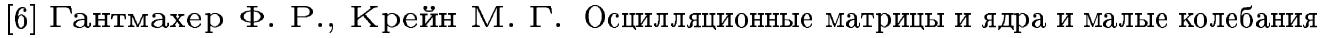
механических систем. М.-Л.: Гостехиздат, 1950. 
[7] Левин А. Ю., Степанов Г. Д. Одномерные краевые задачи с операторами, не понижаюшими числа перемен знака // Сиб. матем. ж. 1976. Т. 17. №3. С. 606-625; Т. 17. №4. C. $813-830$.

[8] Покорный Ю. В.О спектре интерполяционной краевой задачи // УМН. 1977. Т. 32. №6. C. $198-199$.

[9] Покорный Ю. В. О неклассической задаче Валле-Пуссена // Дифференц. уравнения. 1978. T. 14. №6. C. 1018-1027.

[10] Покорный Ю. В., Лазарев К. П. Некоторые осцилляционные теоремы для многоточечных задач // Дифференц. уравнения. 1987. Т. 23. № 4. С. 658-670.

[11] Дерр В. Я. К обобщенной задаче Валле-Пуссена // Дифференц. уравнения. 1987. Т. 23. № 11. С. $1861-1872$.

[12] Покорный Ю. В., Шурупова И. Ю. Об осцилляционных свойствах спектра краевой задачи с функцией Грина, меняющей знак // Укр. матем. ж. 1989. Т. 41. №11. С. 1521-1526.

[13] Тептин А. Л. Об осцилляционности ядра, связанного с функцией Грина одной многоточечной задачи // Дифференц. уравнения. 1990. Т. 26. № 2. С. 358-360.

[14] Боровских А. В., Л азарев К. П., Покорный Ю. В. Об осцилляционных спектральных свойствах разрывных краевых задач // Докл. РАН. 1994. Т. 335. № 4. С. 409-412.

[15] Боровских А. В., Покорный Ю. В. Системы Чебышева-Хаара в теории разрывных ядер Келлога // УМН. 1994. Т. 49. № 3. С. 3-42.

[16] Боровских А. В., Лазарев К. П., Покорный Ю. В. О ядрах Келлога в разрывных задачах // Оптимальное управление и дифференциальные уравнения. Тр. МИАН им. В. А. Стеклова. Т. 211. М.: Наука, 1995. С. 102-120.

[17] Дерр В. Я. О знаке функции Грина обобщенной задачи Валле-Пуссена // Функционально-дифференциальные уравнения. Пермь: ПермПИ, 1986. С. 35-41.

[18] Степанов Г. Д. Эффективные критерии сильной знакорегулярности и осцилляционное свойство функций Грина двухточечных краевых задач // Матем. сб. 1997. Т. 188. № 11. C. $121-159$.

[19] Polya G. On the mean-value theorem corresponding to a given homogeneous differential equation // Trans. Amer. Math. Soc. 1922. V. 24. P. 312-324.

[20] Левин А. Ю. Неосцилляция решений уравнения $x^{(n)}+p_{1}(t) x^{(n-1)}+\cdots+p_{n}(t) x=0 / /$ УМH. 1969. Т. 24. №2. С. 43-96.

[21] Hartman P. On disconjugacy criteria // Proc. Amer. Math. Soc. 1970. V. 24. № 2. P. 374-381.

[22] Полиа Г., Сеге Г. Задачи и теоремы из анализа. Т. II. М.: Наука, 1978.

[23] Калафати П. Д. О функциях Грина обькновенных дифференциальных уравнений // Докл. AH CCCP. 1940. T. 26. №6. C. 535-539. 